\title{
Multidisciplinary Treatment of Advanced Hepatocellular Carcinoma With Severe Arterioportal Shunt: A Case Report.
}

yao-chang luo

The First Affiliated Hospital of guangxi university of Chinese medicine

hai-lin lu (D luhailin89@163.com )

the first affiliated hospital of guangxi medical university

wen-ling song

The First Affiliated Hospital of guangxi university of Chinese medicine

Fei-fei Xuan

Guangxi Medical University

Research Article

Keywords: Multidisciplinary treatment, Hepatocellular carcinoma, Arterioportal shunt

Posted Date: March 31st, 2021

DOI: https://doi.org/10.21203/rs.3.rs-355022/v1

License: (c) (i) This work is licensed under a Creative Commons Attribution 4.0 International License.

Read Full License 


\section{Abstract}

Hepatocellular carcinoma (HCC) is the fourth most lethal malignancy globally. However, most patients present in advanced stages with limited curative treatment options. Therefore, multidisciplinary treatment is often warranted. Here we report a patient with HCC combined with severe arterioportal shunt (APS) who was treated with multidisciplinary approach, comprising interventional radiology procedures, apatinib and camrelizumab. A 31-year-old man who presented to our hospital because of hematemesis caused by HCC with a severe APS. The patient underwent multidisciplinary treatment including transjugular intrahepatic portosystemic shunt, transarterial chemoembolization of arterial branches feeding the shunt and hepatic lesions and subsequent molecular target therapy combined with immunotherapy. After treatment, stable condition of intrahepatic mass and a notable decrease in the number and size of the lung lesions were observed. The patient achieved a long-time survival of more than 2 years. This data suggests that multidisciplinary treatments may be efficacious in the treatment of advanced HCC with severe APS.

\section{Introduction}

Hepatocellular carcinoma is the 4th leading causes of cancer-related death globally[1]. However, most patients are diagnosed as unresectable or advanced stage disease with limited curative treatment options. Therefore, novel therapeutic strategies for unresectable or advanced HCC patients are desperately warranted. In this report, we describe the case of a patient with advanced HCC accompanied with severe intratumoral APS that showed a favorable response following multidisciplinary treatment including interventional treatment, apatinib and camrelizumab.

\section{Case Presentation}

A 31-year-old man who had been suffered from chronic HBV-associated liver cirrhosis for 10 years, had presented to another hospital with hematemesis in June 11,2018. He was then diagnosed as having HCC with a severe intratumoral APS, which caused portal hypertension that lead to esophagogastric varices bleeding (Fig. 1). Thereafter, he underwent endoscopic variceal ligation, followed by endoscopic injection sclerotherapy three times. Although all that was performed, the esophagogastric varices deteriorated further. The patient was then referred to our hospital in November 19, 2018 for further treatment. Magnetic resonance images in our hospital showed similar findings to outside hospital CT. Chest CT scan was not available but chest $\mathrm{X}$-ray examination revealed no abnormality. Preoperative biochemical examinations showed alpha-fetoprotein (AFP) exceeded $2000 \mathrm{ng} / \mathrm{ml}$ (normal range: $0-8 \mathrm{ng} / \mathrm{ml}$ ), which was the upper limit of our laboratory. Hemoglobin was $78 \mathrm{~g} / \mathrm{L}$, total bilirubin was $13.4 \mu \mathrm{mol} / \mathrm{L}$, total protein was $73.6 \mathrm{~g} / \mathrm{L}$, albumin was $41 \mathrm{~g} / \mathrm{dL}$, prothrombin time was $15.5 \mathrm{~s}$, and creatinine was $55 \mu \mathrm{mol} / \mathrm{L}$, respectively. The patient's Child-Pugh score was six points (Class A) and the model for end-stage liver disease score was nine. The patient was classified by the Barcelona Clinical Liver Cancer (BCLC) system as stage $\mathrm{B}$. According to the BCLC treatment algorithms, the patient preferred transarterial chemoembolization (TACE) to surgical resection, radiofrequency ablation, and other therapeutic options. 
Since the severe APS cause portal hypertension that lead to esophagogastric varices bleeding, transjugular intrahepatic portosystemic shunt (TIPS) and APS embolization were performed to reduce risk of rebleeding. Hepatic angiography using a $5 \mathrm{~F}$ catheter revealed a marked APS communication between the left hepatic artery and the left branch of portal vein accompanied with reflux into the main portal vein (Fig. 2). The left hepatic artery was occluded with multiple coils and gelatin sponge through a 2.7 F microcatheter (Progreat, Terumo, Japan) to reduce the blood flow through the APS. Subsequently, TIPS via using two self-expandable stents (FLUCENLY, $8 \times 100 \mathrm{~mm}$ and $8 \times 80 \mathrm{~mm}$ each) was achieved (Fig. 2), followed by embolization of the esophagogastric varices with a mixture composed of histoacryl and iodized oil. The procedure was completed without procedure-related complications. After the procedure, the portal pressure in direct measurements decreased from $50 \mathrm{~cm} \mathrm{H}_{2} \mathrm{O}$ to $40 \mathrm{~cm} \mathrm{H}_{2} \mathrm{O}$.

Despite the TIPS operation, the portal pressure was not well improved. Given this, TACE was performed again to reduce the blood flow through the APS 15 days later. Embolization with coils and polyvinyl alcohol particles were initially performed to occlude the shunt, followed by infusion of a small amount of iodized oil and $10 \mathrm{mg}$ pirarubicin emulsion. After the procedure, repeated hepatic arteriography showed that the APS reduced but existed.

The patient had an uneventful recovery after TIPS and TACE. Eleven days after the TACE procedure, he was discharged from hospital with close follow-up.

The patient was re-hospitalized to undergo TACE on January 22, 2019. Meanwhile, molecular target therapy with apatinib was started. $250 \mathrm{mg}$ apatinib orally once daily. Shortly after the initiation of apatinib treatment, he was suffered from hematemesis (Common Terminology Criteria Adverse Events version 5.0, grade 3). Complete hemostasis was obtained after apatinib therapy was interrupted. About 3 months after the 2nd TACE, CT scans showed enlarged tumors in the liver, with lung metastases, and the serum AFP level increased to $1256 \mathrm{ng} / \mathrm{mL}$.

With careful consideration and the patient's informed consent, we initiated immunotherapy with camrelizumab in August 1, 2019. Camrelizumab 200 mg was administered intravenously over 30 min once every 2 weeks in a 4 week cycle. The patient tolerated the infusion without any complications. After another three cycle of therapy, the follow-up CT scans showed stable hepatic lesions and a continued decrease in the size of the lung lesions (Fig. 3). However, camrelizumab therapy was discontinued due to economy issue.

Subsequently, TIPS revision and 2 sessions of TACE were performed. The latest follow-up CT obtained in September 2020 showed tumor burden decreased, the severe APS relieved and most lung lesions diminished in size or disappeared. However, he passed away 3 months later following the last visit.

\section{Discussion}

In this case, we report a case of advanced HCC with APS undergoing multidisciplinary treatment including interventional treatment, apatinib and camrelizumab. To the best of our knowledge, such case 
has not been previously documented.

HCC combined with APS is a common phenomenon in clinical practice. APS has been reported in 28.8$63.2 \%$ of HCC cases[2]. Severe APS aggravates the complications of portal hypertension, diminish the efficacy and safety of TACE, and even promote lung metastases. Portal hypertension arising from APS leads to rupture of gastroesophageal varices, which is a life-threatening complication in cirrhotic patients with a 6-week mortality as high as $20 \%[3]$. Therefore, it is essential to treat severe APS for these patients. Endovascular embolization remains the treatment of choice. Several studys suggested that TACE with various embolic agents such as coil, ethanol, polyvinyl alcohol, and gelatin sponge is an effective treatment[4, 5]. Herein, we use coil combined with gelatin sponge or polyvinyl alcohol to embolize the severe APS. Subsequently, routine TACE was performed if there were residual tumor feeders after embolization. Unfortunately, APS was relieved but resistant to two TACE procedures. For the treatment of esophagogastric varices bleeding as a result of portal hypertension, endoscopic treatment is the standard treatment and has been tried in multiple studies with some degree of success. However, in our case, endoscopic treatment was usually insufficient to eradicate the varices and fail to control the esophagogastric varices bleeding, while TIPS can be used as a second-line therapy and it was proven to be efficacious and safe in the treatment of esophagogastric varices bleeding[6]. TIPS reduce the hepatic venous pressure gradient, which results in portal venous decompression and variceal bleeding control. In the presented case, we performed TIPS in combination with endovascular embolization of the APS to decrease portal hypertension. After TIPS creation and APS embolization, the portal pressure decreased from $50 \mathrm{~cm} \mathrm{H}_{2} \mathrm{O}$ to $40 \mathrm{~cm} \mathrm{H}_{2} \mathrm{O}$. The patient remained stable for the rest of the hospital course with no signs of recurrent bleeding. The result suggested that our treatment strategy was effective and produced no major complications.

In patients with BCLC stage B, palliative locoregional treatments such as TACE remain the gold standard for therapy. However, it is recommended to initiate systemic therapy if no response is observed after 1 or 2 sessions of TACE, or progression with extrahepatic spread[7]. In the present case, the patient was initially treated with interventional treatment alone. Unfortunately, not only the tumor and the APS were not controlled, but also extrahepatic metastasis emerged. Under this condition, the treatment strategy then to move from locoregional treatment to systemic therapy. Therefore, we performed multidisciplinary treatment including molecular targeted therapy and immunotherapy.

Angiogenesis is mediated by vascular endothelial growth factor (VEGF) and act as an important role in the process of tumor growth, invasion, and metastasis. VEGF combines with VEGF receptor could activate the downstream signals, thereby stimulate the proliferation of vascular endothelium. It is plausible that blockage of VEGFR-2 could be a promising strategy to inhibit tumor induced angiogenesis. Therefore, drugs for the VEGFR anti-angiogenic pathway are being increasingly used for treatment. Apatinib (HENGRUI MEDICINE Co, Ltd. Jiangsu, China) is a novel and highly selective VEGFR-2 tyrosine kinase inhibitor and is the first generation of oral antiangiogenesis drug. Previous studies have suggested that apatinib shows promising clinical efficacy against a variety of solid tumors[8, 9]. Molecular targeted therapy has been a hot topic in multidisciplinary therapy of HCC. However, there is little data about 
apatinib in treating with HCC. For this case, we attempted to administrate him molecular targeted therapy with apatinib. We prescribed him apatinib $250 \mathrm{mg}$ once daily. The patient showed stable condition of intrahepatic mass and a gradual decrease in AFP after apatinib therapy. Unfortunately, he suffered hematemesis and we had to stop the medicine.

Recently, cancer immunotherapies have attracted much attention as potential clinical treatments, particularly for patients with late-stage disease. A major breakthrough was the development of antibodies that targeting negative regulators of T-cell activation, named immune checkpoints. Immune checkpoint therapy mainly includes programmed cell death-ligand 1 (PD-L1) and programmed cell death protein-1 (PD-1) inhibitors. Tumor cells express PD-L1, which interact with PD-1 receptors, which prevent cytotoxic T cells from destroying tumor cells. Immune checkpoint inhibitors restore the cytotoxic T cell's ability to destroy tumor cells by blocking the PD-1 receptor. Moreover, the specific inhibition of the PD-1 checkpoint significantly increased the antitumor efficacy of $T$ cell. Thus, anti-cancer immunity can be enhanced by antibodies that block the PD-1/PD-L1 interaction. Immune checkpoint inhibitors have been extensively studied in multiple tumors, including HCC [10-12]. These trials showed significant potentiality of PD1/PD-L1 inhibitor in treating HCC. Camrelizumab (HENGRUI MEDICINE Co, Ltd. Jiangsu, China) is a monoclonal antibody that inhibits PD-1 immune checkpoint signaling. In a recent trial, 32 of 217 (14.7\%) patients with previously treated advanced HCC experienced objective response with tolerable adverse effects, and the 6-month overall survival probability was 74.4\%[13]. In this case, the patient exhibited a promising clinical response with camrelizumab therapy. After administration of camrelizumab, stable condition of intrahepatic mass, a notable decrease in the number and size of the lung lesions and a significant decline in AFP levels, were observed (Fig. 3 and Fig. 4). We speculated that two or more cycles of camrelizumab treatment was significantly associated with objective response. Considering the promising evidence from the camrelizumab trial study, China National Medical Products Administration officially approved camrelizumab as second-line treatment for advanced HCC patients.

Notably, recent clinical data revealed that immunotherapy combined with molecular target therapy might develop synergetic effects. Lijun Liang et al.[14] reported that patients who received PD-1 blockadeactivated multiple antigen-specific cellular therapy in combination with apatinib showed a significant improvement in progression-free survival and a stronger change in the circulating $T$ cells. Those patients also tended to exhibit longer overall survival, even though the difference was not statistically significant. The promising results may be due to a normalization of the tumor microenvironment. Angiogenesis and immunosuppression constitute the tumor microenvironment, while VEGF/VEGFR pathway take an important role in the regulation of tumor microenvironment immune status. Apart from antiangiogenic properties of apatinib, the inhibition of VEGFR2 also has immunomodulatory effects mediated via reducing the number and function of regulatory $T$ cells and myeloid-derived suppression cells, and enhancing dendritic cells maturation and effector T cells mobilization, activation, and infiltration, thereby resulting in the reprogramming of the immunosuppressive tumor microenvironment into an immunostimulatory microenvironment, ultimately reducing their immunosuppressive effects and suppressing tumor growth. Therefore, although apatinib administration was interrupted in our case, it is reasonable to hypothesize that the tumor microenvironment was changed by apatinib, enabling greater 
responses to the immune checkpoint blockade, as described above. Taken together, synergism between immunotherapy and molecular target therapy forms the rationale for the development of combined camrelizumab and apatinib therapy. Camrelizumab alone or in combination with apatinib may also be a likely first-line treatment option in treating with advanced HCC. However, the expensive cost of camrelizumab with no medical insurance reimbursement limits the utilization.

\section{Conclusions}

HCC is a complex disease that needs a multidisciplinary treatment strategy. The results obtained from our case demonstrate an impressive tumor control from the multidisciplinary treatment in patient with advanced HCC. Multidisciplinary treatments, comprising interventional radiology procedures, molecular target therapy and immunotherapy can be a potent treatment option for advanced HCC accompanied by severe APS, even in cases involving multiple extrahepatic metastases. However, further prospective casecontrol studies are needed to confirm our findings.

\section{Declarations}

\section{Ethical approval}

All procedures performed in this study involving human participants were in accordance with the ethical standards of the institutional and/or national research committee and with the 1964 Declaration of Helsinki and its later amendments or comparable ethical standards.

\section{Consent for publication}

Informed consent was obtained from the patient's wife.

\section{Availability of data and materials}

All data generated or analysed during this study are included in this article.

\section{Competing interests}

All authors declare no conflicts of interest.

\section{Funding source}

This work was supported by the Middle-aged and Young Teachers' Basic Ability Promotion Project of Guangxi (CN) (grant number: 2020KY03014).

Authors' Contributions $Y L$ was the chief physician who took the charge of the patient; $\mathrm{HL}$ wrote the first manuscript; WS did the literature review and followed-up; FX revised the language and grammar of the manuscript. All authors read and approved the final manuscript. 
None.

\section{References}

1. Bray F, Ferlay J, Soerjomataram I, et al. Global cancer statistics 2018: GLOBOCAN estimates of incidence and mortality worldwide for 36 cancers in 185 countries. CA: a cancer journal for clinicians 2018, 68(6):394-424.

2. Oh D, Shin SW, Park HC, et al. Changes in arterioportal shunts in hepatocellular carcinoma patients with portal vein thrombosis who were treated with chemoembolization followed by radiotherapy. Cancer research and treatment : official journal of Korean Cancer Association 2015, 47(2):251-258.

3. Deltenre P, Trépo E, Rudler M, et al. Early transjugular intrahepatic portosystemic shunt in cirrhotic patients with acute variceal bleeding: a systematic review and meta-analysis of controlled trials. European journal of gastroenterology \& hepatology 2015, 27(9):e1-9.

4. Kim YJ, Lee HG, Park JM, et al. Polyvinyl alcohol embolization adjuvant to oily chemoembolization in advanced hepatocellular carcinoma with arterioportal shunts. Korean journal of radiology 2007 , 8(4):311-319.

5. Chan WS, Poon WL, Cho DH, et al. Transcatheter embolisation of intrahepatic arteriovenous shunts in patients with hepatocellular carcinoma. Hong Kong medical journal = Xianggang yi xue za zhi 2010, 16(1):48-55.

6. Carey W. Portal hypertension: diagnosis and management with particular reference to variceal hemorrhage. Journal of digestive diseases 2011, 12(1):25-32.

7. Wörns MA, Galle PR. Hepatocellular carcinoma in 2017: Two large steps forward, one small step back. Nature reviews Gastroenterology \& hepatology 2018, 15(2):74-76.

8. Li J, Qin S, Xu J, et al. Randomized, Double-Blind, Placebo-Controlled Phase III Trial of Apatinib in Patients With Chemotherapy-Refractory Advanced or Metastatic Adenocarcinoma of the Stomach or Gastroesophageal Junction. Journal of clinical oncology : official journal of the American Society of Clinical Oncology 2016, 34(13):1448-1454.

9. Peng $\mathrm{H}$, Zhang $\mathrm{Q}$, Li J, et al. Apatinib inhibits VEGF signaling and promotes apoptosis in intrahepatic cholangiocarcinoma. Oncotarget 2016, 7(13):17220-17229.

10. Heymach J, Krilov L, Alberg A, et al. Clinical Cancer Advances 2018: Annual Report on Progress Against Cancer From the American Society of Clinical Oncology. Journal of clinical oncology : official journal of the American Society of Clinical Oncology 2018, 36(10):1020-1044.

11. Zhu AX, Finn RS, Edeline J, et al. Pembrolizumab in patients with advanced hepatocellular carcinoma previously treated with sorafenib (KEYNOTE-224): a non-randomised, open-label phase 2 trial. The Lancet Oncology 2018, 19(7):940-952.

12. El-Khoueiry AB, Sangro B, Yau T, et al. Nivolumab in patients with advanced hepatocellular carcinoma (CheckMate 040): an open-label, non-comparative, phase 1/2 dose escalation and 
expansion trial. Lancet (London, England) 2017, 389(10088):2492-2502.

13. Qin S, Ren Z, Meng Z, et al. Camrelizumab in patients with previously treated advanced hepatocellular carcinoma: a multicentre, open-label, parallel-group, randomised, phase 2 trial. The Lancet Oncology 2020, 21(4):571-580.

14. Liang L, Wen Y, Hu R, Wang L, Xia Y, Hu C, Qiao Y, Geng X, Chen T, Fei J et al: Safety and efficacy of PD-1 blockade-activated multiple antigen-specific cellular therapy alone or in combination with apatinib in patients with advanced solid tumors: a pooled analysis of two prospective trials. Cancer immunology, immunotherapy : CII 2019, 68(9):1467-1477.

\section{Figures}
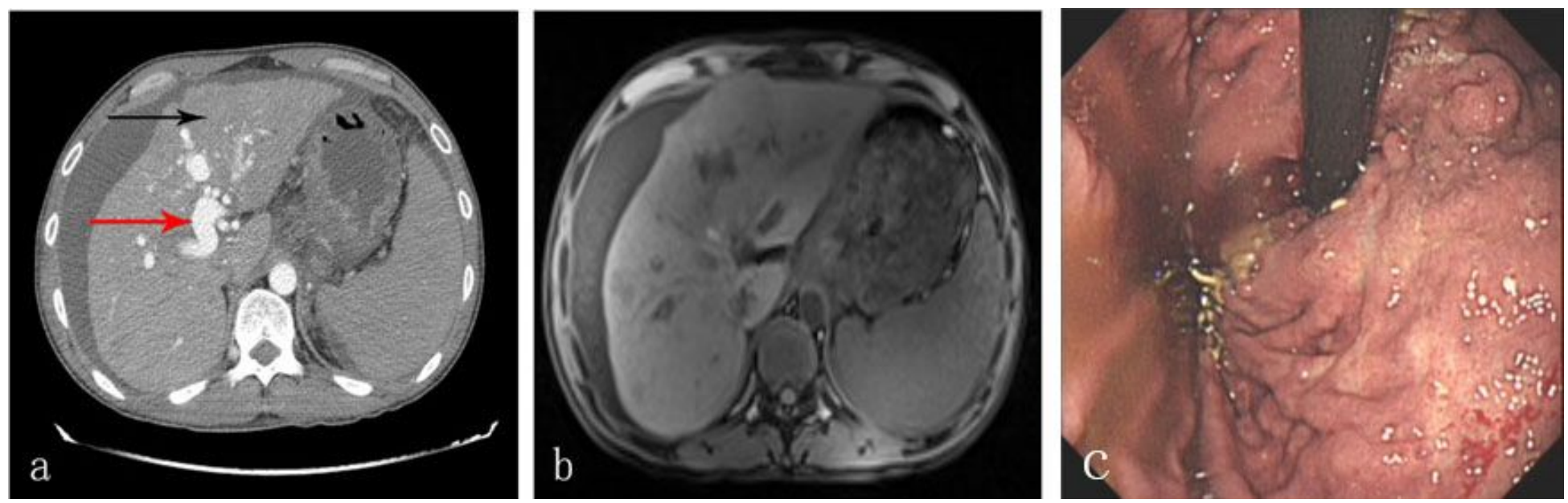

\section{Figure 1}

Contrast-enhanced computed tomography (CT), Magnetic resonance images (MRI) and upper gastrointestinal endoscopy findings. (a) CT showed a huge mass measuring approximately $7 \mathrm{~cm}$ in diameter in the left hepatic lobe (black arrow), with daughter nodules in the right hepatic lobe. The lesion is unenhanced at the arterial phase because the tumorous APS reduces arterial flow to it. Hyper-enhanced portal vein was present in arterial phase CT image (red arrow). CT images also identified ascites and splenomegaly. (b) MRI in our hospital showed similar findings to outside hospital CT. (c) Upper gastrointestinal endoscopy revealed severe esophagogastric varices. 

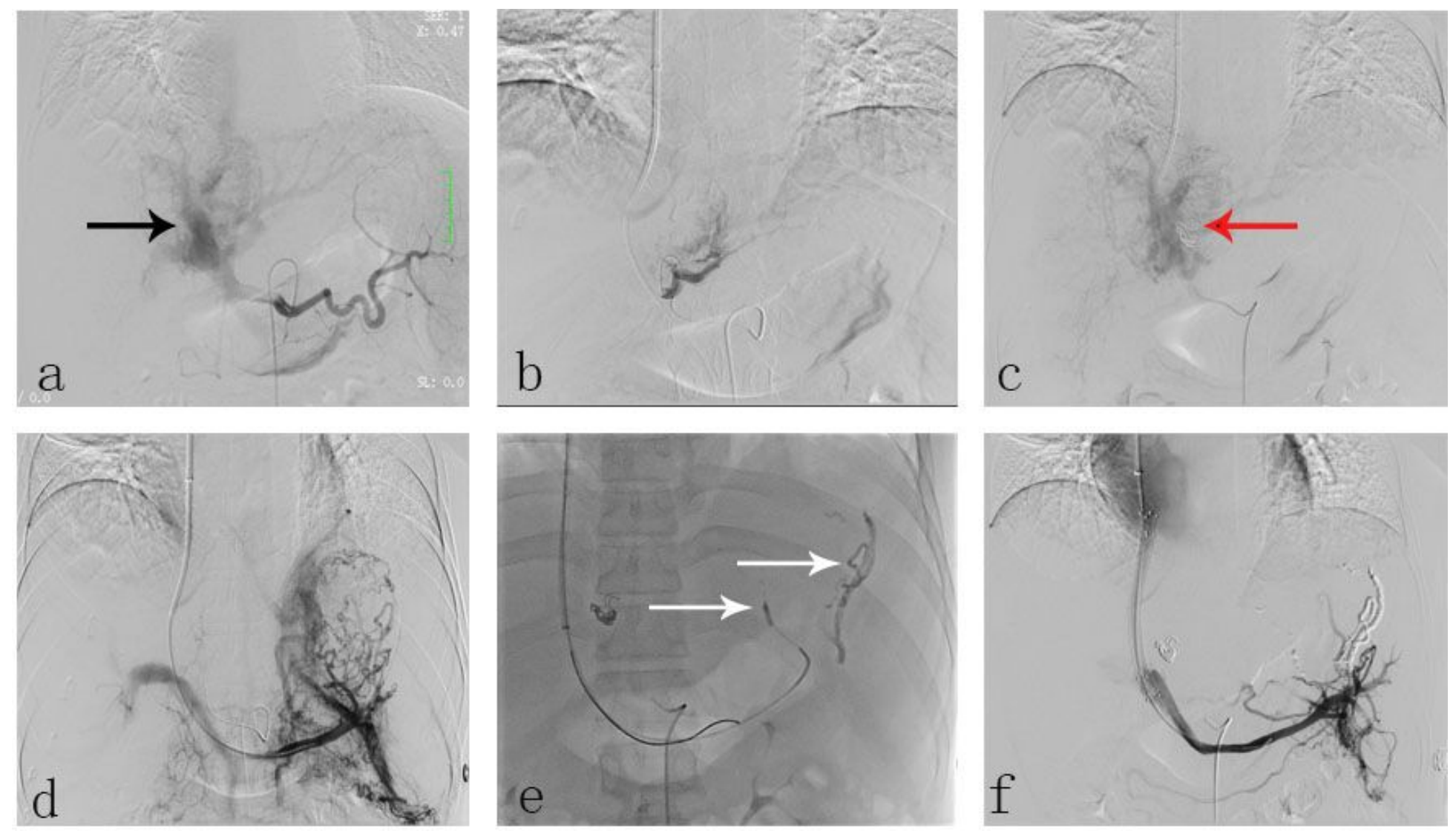

\section{Figure 2}

(a) Hepatic angiography shows a marked APS communication between the left hepatic artery and the left branch of portal vein accompanied with reflux into the main portal vein (black arrow). (b) Left hepatic artery angiogram demonstrates the tumor staining. The tip of the microcatheter is placed in a feeding artery. (c) Post-embolization shows partly occlusion of the APS by multiple coils (red arrow) and gelatin sponge. (d) Major findings of portal vein angiography during TIPS. (e) Embolization of the gastric varices with a mixture composed of histoacryl and iodized oil (white arrow). (f) Portogram obtained immediately after TIPS creation shows good passage of contrast medium through the shunt. 

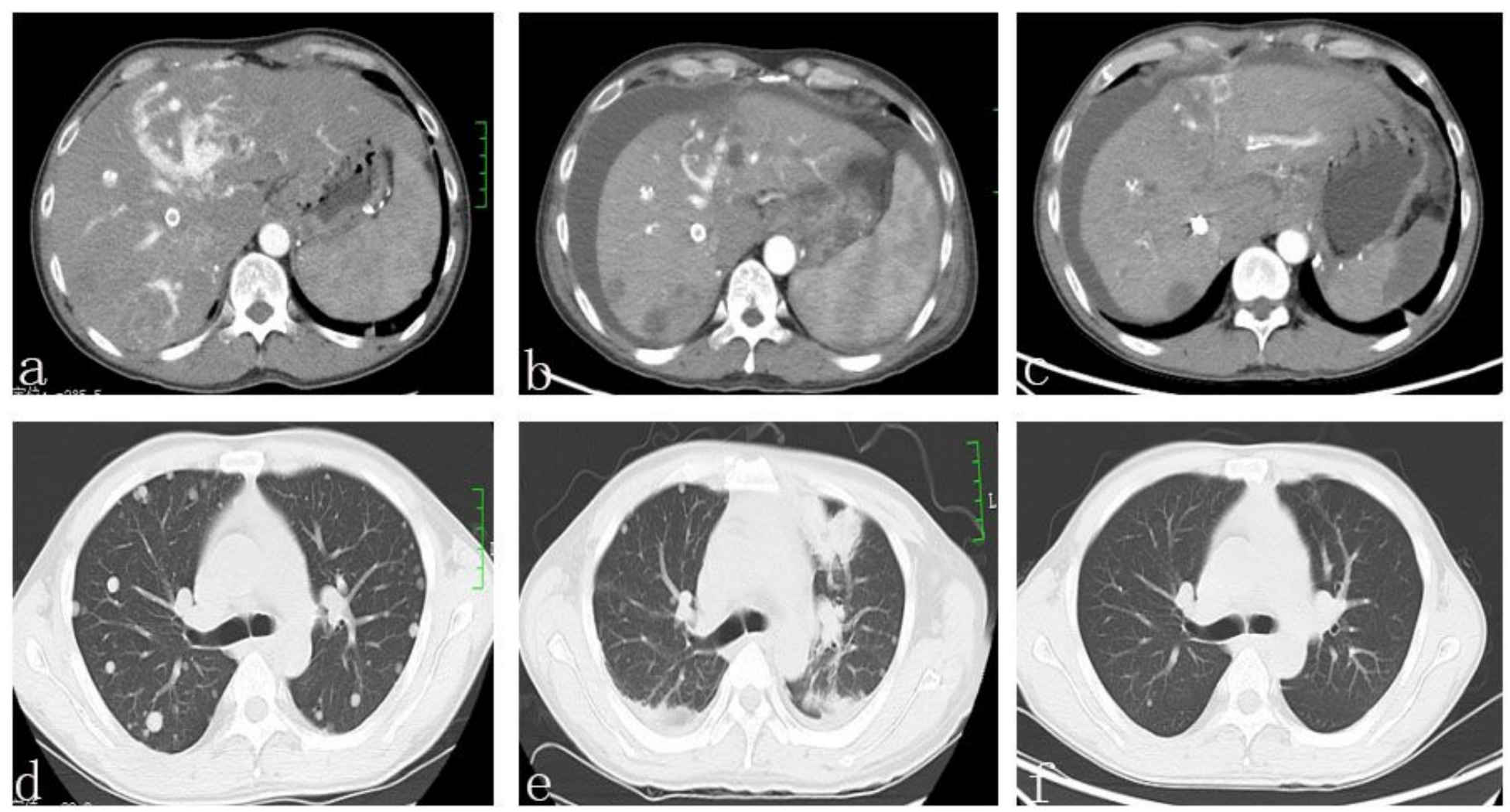

\section{Figure 3}

Contrast-enhanced computed tomography before and after camrelizumab therapy. CT images before treatment with camrelizumab (a,d) and after two cycles (b, e) and four cycles (c, f). CT image shows HCC with APS and multiple bilateral lung metastases $(a, d)$. All lung lesions rapidly responded to camrelizumab (b, e), and after four cycles, most lesions were diminished in size or disappeared. Liver masses remained stable and APS relieved $(c, f)$. 


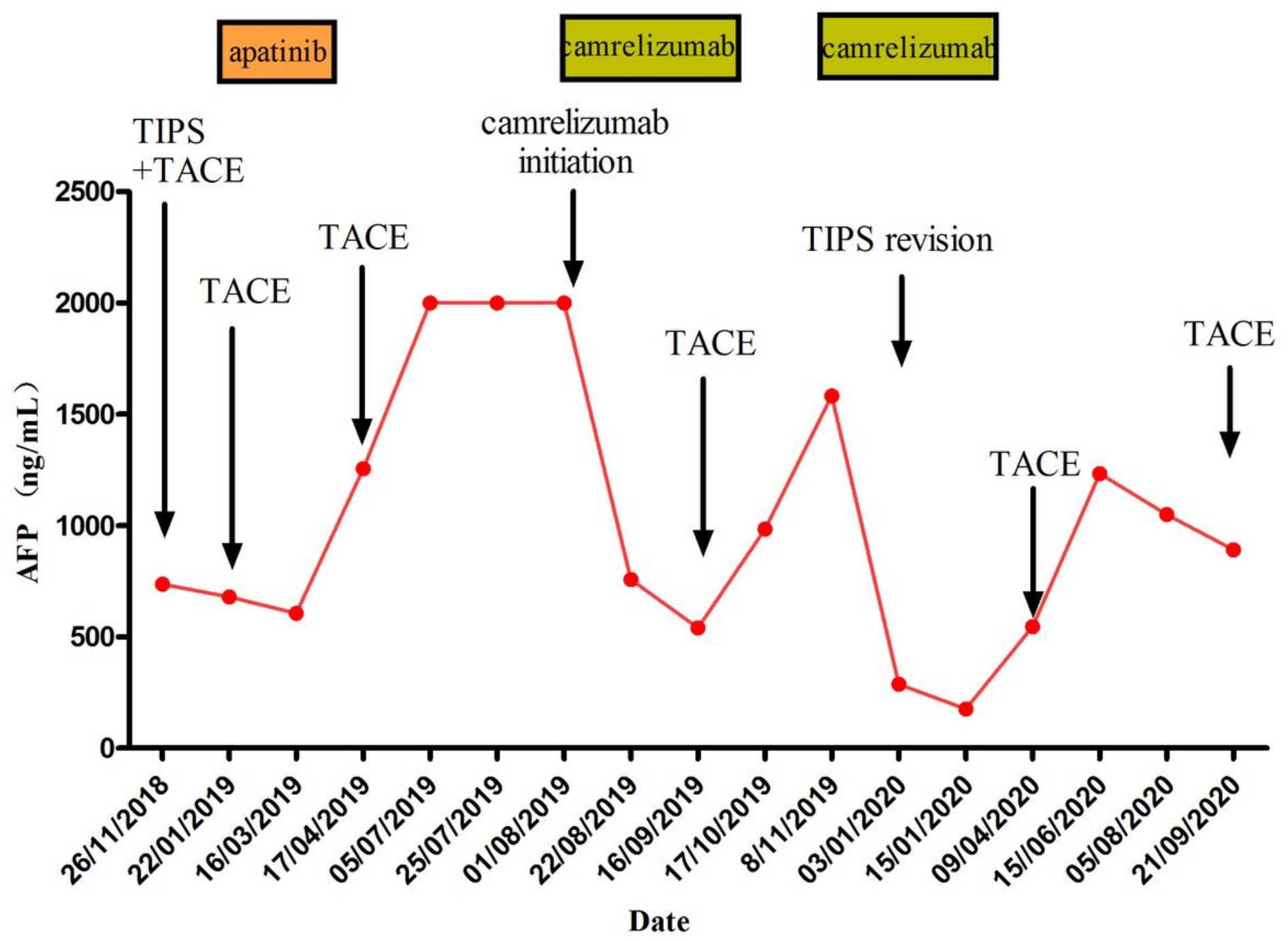

Change in AFP level during the course of therapy

Figure 4

Clinical course as assessed by AFP and therapeutic events. 